\title{
Toward an Adaptive Enterprise Modelling Platform
}

\author{
Amjad Fayoumi \\ Management Science, Lancaster University, Lancaster LA1 4YX, UK \\ a.fayoumi@lancaster.ac.uk
}

\begin{abstract}
For the past three decades, enterprise modelling (EM) has been emerging as a significant yet complex paradigm to tackle holistic systematic enterprise analysis and design. With a high fluctuation in the global economy, industrial stability and technology shift, the necessity of such paradigms becomes crucial in determining the decisions that an enterprise can make for surviving in such a highly dynamic business ecosystem. EM practices have focused for a long time, on the design-time of enterprise systems. Recently, there has been a rapid development in data analytics, machine learning and intelligent systems from which an EM platform can benefit. EM needs to cope with the new changes in both business and technology; it should also help architects to determine optimum decisions and reduce complexity in technical infrastructure. In this paper, the author discusses several challenges facing enterprise modelling practices and offers an architectural notion for future development focusing on the requirements of a platform that can be called intelligent and adaptive.
\end{abstract}

Keywords: Enterprise Modelling, Enterprise Modelling Challenges, Enterprise Modelling Adaptive Platform.

\section{Introduction}

In recent years, we have seen a rapid advancement in practices and technologies that aid enterprise development and their ability to support informed and timely decisions. Enterprise Modelling (EM), like many other practices in information systems (IS) research, has seen much interest and development, and in fact, has proved useful for many enterprises in the industry. Although EM has developed significantly in the last three decades and helped organisations in their business and IT (Information Technology) transformation efforts, it still needs to incorporate the paradigm shift in technology. Recent researches in the area of EM have highlighted the need to increase the sophistication and capabilities of both their practices and tools as they are still far from their maximum potential [1]. For instance, previous researches have acknowledged the issues of integration and interoperability of enterprise models, and organizations' need to be able to exchange and integrate their enterprise models easily [2]. In addition, specific research has suggested further consideration of assistive technology [1]. EM has for a long time been concerned with the enterprise designtime; the focus should also cover the run-time. Previous researches almost neglected 
the paradigm shift but now organisations are moving towards more shared service models that heavily use APIs and micro-services towards more data analytics and more automation that reduces human errors and minimises their involvement in the Cyber-Physical Systems (CPS)[3]. EM practices should demonstrate how enterprises can be designed for future demand, increased resilience, agility and be able to respond to emergent changes rapidly.

This paper argues that future EM can support this notion through the use of intelligent and knowledge-based systems toward an adaptive enterprise modelling platform. What is meant by adaptive EM platform is bringing together the two notions of automated design, and run-times enterprise models intertwined, thus enabling the design, governance and validation of enterprise models within the same platform. We propose using advanced analytics and AI (Artificial Intelligence), to enable selfhealing or the correction mechanism enterprise systems that are performed by systems instead of humans $[4,5]$. In this case, the role of the enterprise designer is limited to monitoring the dashboards to check the enterprise status and to recreate only if adjustment is required for any part of the enterprise models. With a versioning mechanism, we can also track the evolution of the enterprise models, how it was and what it became with the help of intelligent systems. This paper focuses on the following research questions:

1. What are the current themes of EM and the focus of current research?

2. What are the limitations and challenges of EM research and practices?

3. What are the requirements for an adaptive enterprise modelling platform?

4. How possible is it to implement this platform through utilising available tools?

The rest of the paper is structured accordingly: Section 2 offers a general review on EM practices and illustrates the current themes and capabilities of contemporary EM frameworks. There is then discussion of the current challenges of these practices from an IT paradigm shift perspective toward more adaptive and intelligent platforms. Section 3 identifies future EM platform requirements for an intelligent and adaptive EM platform, and offers a notion on how it can be implemented. Finally, the paper concludes with Section 4, which discusses the next steps of this research.

\section{Enterprise Modelling and Architecture}

\subsection{Historical Background}

Conceptual modelling (CM) became a basis for enterprise modelling [6-8], simulation [9], and information systems development [10-12]. In information systems research $\mathrm{CM}$ is used to describe behavioural, structural, informational or functional aspects of a system. The system is socio-technical and involves organisation systems and software systems. For software systems development, the CM helped: 1) to generate code from conceptual models through model-driven development, to build a software application [13], 2) the logical formulation of these models using formal logic languages to prove and validate the models mathematically, and 3) to complement models with algorithms and equations for quantitative and statistical representation of 
data parameters. The applications of these techniques are numerous and can be applied to any aspect or level of the enterprise architecture. Formal conceptual modelling can be described visually by using syntactically and semantically-sound graphical notations.

CM began in the early days of software engineering practice, particularly with the development of the relational model in the early 1970s [14]. The main purpose of CM in that time was to better represent software domain requirements, with the intention of designing valid and verified software systems [11]. CM has continued to improve and has extended beyond its traditional scope of software engineering to cover areas such as business strategy and operation $[6,9,15]$. Elsewhere, strategic information system practices were developed to guide and structure IS procurement, development, and deployment in organizations. One of the other reasons that strategic information system practices were developed was to improve the value generation and alignment between business and IT $[16,17]$. The use of CM to aid both business and IT strategies, and the alignment between them, resulted in the developing area of the enterprise architecture (EA) framework [18], where EM tools play a major role in visually presenting enterprise architecture artefacts. Some of the well known EA frameworks are: Zachman [19], TOGAF [20], and DoDaF/MoDaF [21].

\subsection{Current Themes and Capabilities in EM Research}

The recent developments in EM research look promising. One of the focuses of a recent development was the domain-specific modelling languages [22, 23], which can offer models (syntax, semantic, and notations) embedded in modelling tools for specific business or system domains. Loucopoulos, Stratigaki [24] introduced capability oriented enterprise modelling, focusing on the concept of capability and how it responds to an enterprise and changing needs [25]. Fill [26] developed a modelling framework from semantic annotation called SeMFIS (Semantic-based Modelling Framework for Information Systems). Multi-perspective Enterprise Modelling (MEMO) also shows a sophisticated development in terms of metamodel, notations and enterprise aspects integration. These frameworks were implemented using the ADOxx framework [27]. Boissier, Rychkova [28] proposed an extension of the EM practices for a decentralised enterprise, e.g. corporate and holding companies, with the model containing a metamodel and practices for tackling enterprise efforts in a similar environment. Hinkelmann, Gerber [16] also proposed an approach using the metamodelling framework ADOxx [29] and integration ontology to align business with IT. The same framework was used for creating domain-specific modelling languages [30]. Many of these initiatives were part of OMiLAB [29] - the Open Model initiative Laboratory. Two other important EM frameworks are DEMO (e.g. Dietz [31]) and 4EM (e.g. Sandkuhl et al. [32]). Another area which has also received attention is that which is relevant to architecture patterns [33], whereby an analyst can orchestrate enterprise models from previously defined patterns, and thus speed up the modelling process and deployment [10]. Patterns can be structural, behavioural, functional, constraining, viewpoints-related, or can be a value proposition. These patterns can be used and re-used in different scenarios within different organizations. 
Another line of recent research focuses on simulation. One interesting implementation is related to the effort made to map business-process modelling notations to simulation-executable specifications. The Workflow Management Coalition (WfMC) has developed a standard, called BPSim, to respond to the need to support interoperability between modelling standards and simulation engines. BPSim can interchange and parameterize business-process analysis data to apply KPIs better, predict business performance, validate process design, allocate resources, and reduce overall operational risk [34]. Simulation can cover both discrete-event [35] and continuous dynamic [10] simulations. Other simulation techniques such as agentbased simulation, graph transformation, and network analysis can be used for different simulation purposes. Other recent researches have also made a considerable contribution in linking both Business Process Modelling and Notation (BPMN) and process mining [36]; further consideration of this mechanism is given in our suggested platform. A summary describing the capabilities of current enterprise modelling platforms and frameworks are presented as the following:

- Modelling notation: A graphical representation that has sound syntax, sometimes it is supported by a procedural approach to guide the designer through the modelling steps. It is used to model and simulate both the current enterprise state ('as is') and the future design goal state ('to be'). The design stage may also involve testing, evaluation of the designed model, and the measure of scalability, robustness, agility, and security. Modelling notations can be developed using modelling frameworks such as ADOxx and EMF.

- Interoperability semantic metamodel: This consists of two parts - model integration and model transformation. The integration will allow models from different enterprise perspectives to be linked together semantically; while transformation, with the support of ontology, can help to map model artefacts to another form, which can be used in another model that has a different level of granularity, mathematical formulation, or software code. The metamodel layer should be agile in a way that allows practitioners to change some of its parameters without affecting the entire metamodel semantic. Metamodels can be developed using metamodels editors like MetaEdit, ADOxx and EMF.

- Simulation and logical formulation: To enable simulation and optimization, the model artefacts need to be calibrated to formal logic and mathematical equations. The formal logic describes how the model artefacts are connected to the simulation constructs, and the impact they have on each other. Moreover, depending on the simulation technique used, it might allow for simulating and testing different 'what-if' scenarios and the values-flow between model elements.

- Implementation and code-generating: A typical model-driven development mechanism translates models to code. Here, some of the models should be translated to some sort of software enabler form; from the high-level abstract domain notations rather than building a large number of Unified Modelling Language (UML) analysis and design models. Typically, any of the model driven modelling tools can generate code out of software models e.g. Eclipse, Papyrus, Sparx EA and many others. One of the main challenges here is related to the ability of mapping to serve different levels of implementation scenarios. Pattern orchestration among different levels of model granularity can help to streamline 
the process of scenario change in order to generate different sets of softwaredependent components or code. It is important to mention that not all of the enterprise models are developed for this purpose, rather they capture enterprise holistic knowledge for various purposes.

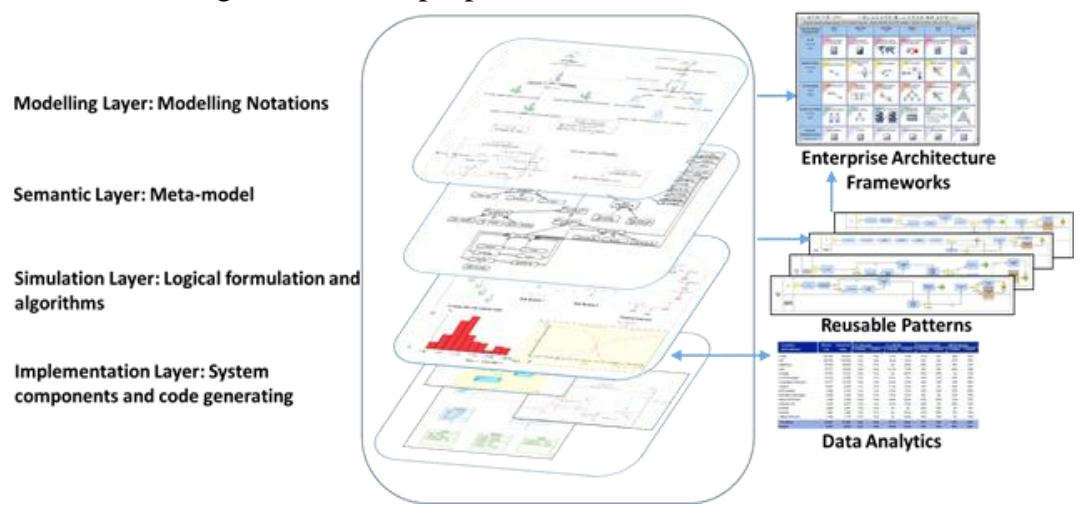

Figure 1. Enterprise modelling and simulation capabilities layers

With reference to Figure 1, the capabilities are recognised as layers of design interface (syntax), design interoperability (semantic), simulation, and implementation. There is feedback from the simulation layer to the design layer, where the simulation of business activities beside advanced data analytics can offer an insight to how enterprise aspects can be designed better to continuously move from 'as is' to 'to be'. Also, it helps in evaluating the current business activities based on performance metrics, and against the design objectives. This, with the support of enterprise simulation and optimization, will feed into rethinking and evolving the architecture and the design models. In the same way, technology and information systems will feed back to business activities in terms of potential new capabilities that can lead to innovation in the applicable business model, and can also provide information about the challenges and limitations that technology imposes on the enterprise's business activities. Thus, technology and information systems might also require a new design, modernization, or optimization. Therefore, feedback regarding modelling, design and simulation is necessary to support appropriate rethinking of the technical design and architecture.

The semantic layer will support the interoperability between modelling notations and simulation engines. Two solutions were proposed in the literature [2] to address models' interoperability: 1) building a unified semantic metamodel that can be used by every tool and every model, and 2) model transformation by building transformation rules to translate between two models, which also requires the use of ontology to map concepts from two different models. The model integration can also take place by both mapping and unifying the model artefacts' semantics. Also, current literature describing the state-of-the-art in the modelling domain has acknowledged that using design and architecture patterns will certainly make the modelling easier. Analysts will be able to orchestrate their enterprise models from previously defined patterns, and thus speed up the modelling process and deployment. Patterns can be 
structural, behavioural, constraining, or values. Business and IS designs should be available in the design process in a repository to cut the design and development time significantly. Also, these patterns can be used and re-used in different scenarios within different organizations. Further, we acknowledge that the analyst/architect experience plays an important role, which impacts the quality of the analysis and design.

\subsection{Challenges to Current Enterprise Modelling Practices}

Despite the long time that EM and EA have been developing, the level of maturity reached in some cases has not met the expectations of some current enterprises. Many projects are subject to failure, or sometimes organizations are not able to fully realize their benefits [37]. Industries are aware of the challenges of adopting EM practices; they must have expert designers, long-term initiative, a long time to perceive sufficient ROI and most of all, the high possibility of failure. Many enterprises find challenges in how to configure their enterprise operations better and effectively align it to enterprise strategy, especially when these practices are adopted by small enterprises who have limited resources [38]. To overcome these challenges, EM practices need to address the following limitations:

- Most of the effort falls on the analyst/designer to decide what needs to be addressed in the enterprise's concerns and to fulfil their objectives of undertaking the entire modelling effort. This needs to be changed to minimise human error and any lack of judgement. More automation and intelligence need to be embedded in the EM system to support decision-making.

- The difficulty of managing and coordinating knowledge among stakeholders from one side and the systems' ever-increasing complexity from the other. It should enable acquiring and exposing information whenever it is required in rigid visualization [39].

- Although EM was presented with the aim of reducing analysis and design complexity, the maintenance and manual updating of enterprise knowledge is still the main theme of how EM is conducted. The current techniques and models have only mitigated this by building domain-specific modelling languages (DSML) [22, 23] which can simplify manual updating for non-expert users. Nevertheless, building domain graphical notations is still an important aspect, but the future development should focus on building an adaptive and intelligent platform that minimises human involvement and relies more on automated decision-making. The EM platform should have the ability to sense and reconfigure enterprise models according to any changes in the environment.

\section{Requirements and Future EM Adaptive Platform}

This section describes the requirements and conceptual model of the target EM adaptive platform as an EM ecosystem, which can help the enterprise be designed for future business requirements within its context, to have increased resilience, and to respond to emergent changes. 


\subsection{Requirements for Adaptive Enterprise Modelling Platform}

Recently, new research has focused on the reverse design that focuses on understanding the enterprise design from the data, e.g. the process mining approach presented in [40]. This work focuses on visualising the process model from a logevents analysis, with an aim to understand what is actually happening when the process is executed and helps to identify any bottlenecks in the process. It also helps in identifying the gap between the actual processes (in run-time) and the designed ones (in design-time). A similar notion is fairly well-developed in tools like 'IBM business process management' [41]; this system has a workflow engine underlying the process model that allows tracking of all the activities and outputs during the runtime with sophisticated dashboards. Such a notion is not widely developed in the mainstream enterprise modelling tools. Some other researches have explored the link between business processes and intelligent systems [42], showing how a role activity diagram (RAD) can be implemented using multi-agent systems.

The adaptive EM platform allows enterprises to intertwine between the design-time and run-time configuration in a semi-automated manner. The platform should enable reconfiguration of the enterprise models according to a set of high-level rules that use advance data analytics and machine learning to visualise models from run-time, and consequently govern, identify gaps, alert and rebuild enterprise models to achieve the goals in the highest enterprise level. To fulfil this aim we identified the set of requirements listed below:

REQ1: Modelling Decision-Support: The EM platform should offer decisionsupport capabilities for enterprise analysts and designers. For instance, designers will select the enterprise business domain, then the reference architecture will be automatically selected to match the selected business domain. The EM platform will ask for the size of the enterprise, number of employees, customers types and segments, products and services. Then the platform will be able to reconfigure the architecture accordingly and suggest core and secondary operational processes with the industrial best practices (e.g. industrial practices listed in [10]) that are required to ensure operational process quality. The system will notify designers about what happens if either a core or secondary process is neglected and thus determines the impact on the enterprise. The system then will suggest what underlying IS services and components are required to execute the processes; offering alternative implementations where possible.

REQ2: The Use of Data Analytics: Data analysis and pattern recognition: Modern enterprise modelling should respond to changes in the enterprise environment. Nowadays, the means of external and internal data collection are increasing. Capturing data and events from numerous enterprise activities and sources such as social, economic, organizational, and financial data can be invaluable to inform the enterprise modelling design. The integration with enterprise systems is crucial and whilst most organizations have already implemented several business applications and enterprise systems, it is important to investigate how designing future enterprise models can make use of the massive amount of data available to be able to inform businesses and IS design. For example, ERP, asset management, and 
supply chain and inventory management systems, can provide input to enterprise modelling. For example, we can generate the organization structure ('as is') and staff profiles from an HRM system; we can also understand the map of the supply chain from an SCM system or understand the current organization and financial divisions from ERP. Another example is the automatic finding of IT infrastructure using asset management systems, which will feed back to the enterprise models, to inform the design system of the available IT capabilities and to show how they can be utilised better. It might offer automated updating of some of the enterprise models once needed. The data can also help in evaluating the efficiency and effectiveness of the designed enterprise models for better optimization (using the simulation models). Finally, the data can be used to create predictive analysis and should support the design of future enterprise models.

REQ3: Intelligent Adaptive System: EM must be more intelligent and proactive. A large number of activities and decisions can be automated to improve responsiveness and minimise errors. Insight from other artificial intelligence (AI) research areas is required (e.g. multi-agent systems, machine learning, knowledge query, and reasoning and rule-based systems) to enhance the responsiveness and adaptability of the enterprise information systems. For example, machine learning can be used for predictive analysis and inform the designer when some aspects of the design need to be changed. Other methods can also be used to support automatic configuration such as game theory, goal-orientated multi-agent systems, and swarm intelligence. Methods inspired by self-healing systems can be used to ensure the stability of the enterprise system.

\subsection{EM for Adaptive Enterprise Systems - Future Scope}

The main goal of developing adaptive systems is to create an autonomic heterogeneous system that can sustainably design and reconfigure itself to handle different types of change and new knowledge [43]. It should consider different types of knowledge that the enterprise ecosystem can offer. It is strongly influenced by evolutionary theory and sees the enterprise as a self-organising entity. Research in the area of adaptive socio-technical systems [43] suggests a need to develop a sociotechnical system framework that can reconfigure itself and evolve within its context by continuously adapting to new requirements over time. To realise this modelling ecosystem, the proposal in this paper focuses on four main components of the adaptive enterprise modelling platform:

1. The design-time: this contains the basic elements of EM, modelling notation, semantic-metamodel, the logical formulation of the models and the simulation engine. The simulation engine can optimise and confirm simulation results toward some specific configuration. Lessons can be learned from process mining methods [40] which extract and visualise processes from operating systems in real time, which in turn helps to support decision-making and perform enterprise transformation or change. The optimiser should be connected to the process-mining visualiser to import real-time data/event-logs to support the design process. The platform will take into consideration the transformation of: a) the current enterprise 
business design to a ubiquitous architecture, and b) the involvement of stakeholders and their impact on the evolution of the entire enterprise. Also, it should be supported by a repository of enterprise models' patterns for quicker deployment and adaptation. The repository will offer the means to extract enterprise systems patterns from legacy systems 'bottom up' which answers the question of what an enterprise can do with its current IS capabilities, and will be able to suggest alternative enterprise systems patterns in a lower level of granularity to implement higher-level enterprise goals 'top down'. One of the other uses of models repositories is that it can foster what is called 'models economy' where experts can generate values when they sell successful, standard-based models. At the execution level, the patterns will be executed using a workflow engine, business rule management systems (rule engines), an events handler, and with the service's code generating in the run-time environment.

2. Decision-support: to offer the logical and automated rules that help designers in constructing enterprise models in responding to Req. 1. The models should correspond to standard practices in industry - this is referred to as domain-specific profiles, and these can be industry-based, e.g. manufacturing or public services, or functional-based, e.g. IT services delivery. When models in a high-level of enterprise granularity are constructed, the platform will suggest what models are required in the lower-level of granularity and which ones are best used with the constructed models. Also, it will support some sort of data analytics and visualisation to analyse the risk associated with deploying one or more enterprise models. This is also supported by real-time data analysis which is required to identify the nature, level and impact of changes and offer feedback to the designer. This is particularly important when information/knowledge is limited, human must intervene to make a decision as human-in-the-loop.

3. Data pool: is needed to address Req. 2, where an organisation gathers all the relevant data or has access to external data which is useful for their business. Both structured and non-structured data is currently stored, and enterprises start to make better decisions by analysing this data using different mechanisms. It is recommended to ingrate the data pipeline with the enterprise design either for direct analysis and visualisation for human decision-making, or to reconfigure and re-link enterprise models, or to change the configuration of the enterprise systems according to a set of predefined rules.

4. The run-time: intelligent systems are required to build an intelligent information system infrastructure [44] that address Req. 3. The run-time of the suggested adaptive platform could help in automating the knowledge or data acquisition into the enterprise information systems' architecture. The run-time will also use techniques of machine learning to handle the acquired data. Therefore, it will adjust the deployment of the run-time using a classifier to classify the acquired knowledge classes and their potential impacts. A synthesizer will work to match the classified data with their relevant enterprise systems and behavioural rules to enable the automatic configuration of one or more parts of the enterprise system. The required change can support: a) optimisation, b) a change in execution rules, events or workflow, and c) a change in the APIs or the software service architecture. Any 
required change in the IS infrastructure will need an involvement from the designer. Furthermore, agents can learn and make decisions towards the optimal goal set by managers using AI techniques which enables agents to evolve, adapt and change their behaviour according to the new situation, in order to achieve the assigned goal (e.g. using human cognitive BDI agent structure (belief, desire, intention, and action))[42]. It will make enterprises adaptable according to the environment changes towards the realisation of the dynamic information systems' architecture. The software agents will interact with each other in a multi-agent system framework. The software agents will also interact with the human actors to audit and control the human behaviour to ensure quality and achievement of the goal. Also, agents will bridge the knowledge from the environment with both the design and run-time platforms. Figure 2 depicts the future adaptive platform components.

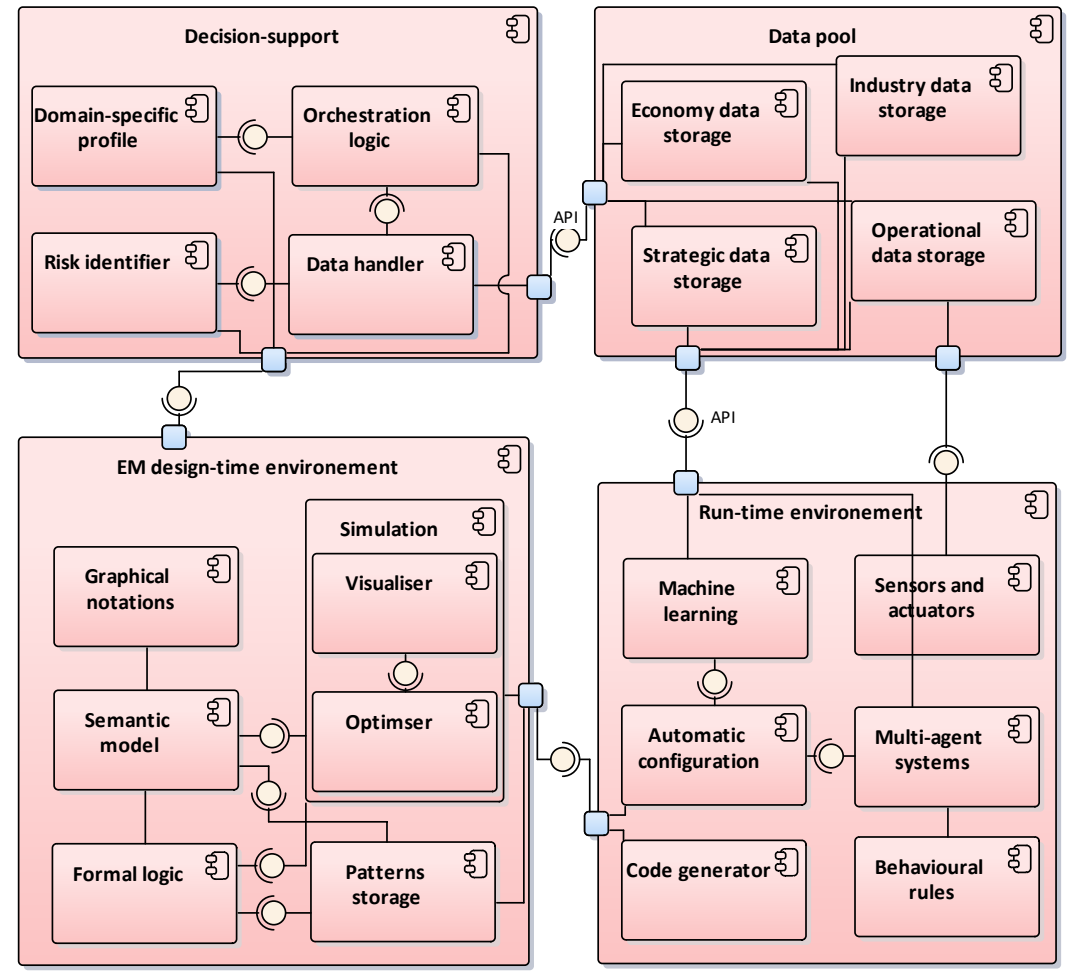

Figure 2. Components for adaptive enterprise modelling platform (future scope)

\section{Conclusions}

Traditional enterprise modelling practices have been challenged by the immense scale of changes in the economy, industry and technology, to which enterprises have been forced to react to in order to deal with past, present, and future decisions. In this paper, we offered a discussion on the current state-of-the-art in EM and discussed the 
limitations and challenges of EM practices. We then presented what is needed for the future EM platform; a list of requirements has been identified. A notion to move forward towards adaptive EM for implementing a next-generation EM platform was also presented. The platform contains components of advanced data analytics, process mining, machine learning and multi-agent systems as additional elements that extend EM capabilities. The research-in-progress presented in this paper follows the design science approach for information systems research [45] by identifying the problem, objectives of the solution and designing the solution. The research will continue in the development of the suggested platform. Future research can focus on developing and implementing the suggested platform by exploiting the successful ADOxx [46, 47] and create an extension of the current ADOxx metamodel. The extension will consider creating a metamodel for both the decision-support components and the intelligent adaptive components. The extension will focus on three aspects relevant to each one of the requirements: 1) a metamodel for decision-support, 2) a metamodel for adaptive run-time, and 3) a metamodel for data analytics and handling; all integrated together with the ADOxx metamodel. Alternatively, the platform can be developed using the Eclipse Modelling Framework (EMF) [48], which can offer an easy integration to a multi-agent systems library, however, it will be challenged by the degree of dynamism that can offers. Then, an evaluation of the developed platform can take place using a credible design science evaluation method, predominantly using case studies and industry-based evaluation. The platform is anticipated to contribute in both academic state-of-the-art and industrial practices.

\section{References}

1. Sandkuhl, K., et al., From Expert Discipline to Common Practice: A Vision and Research Agenda for Extending the Reach of Enterprise Modeling. Business \& Information Systems Engineering, 2018. 60(1): p. 69-80.

2. Karagiannis, D., et al., Metamodeling: Some Application Areas in Information Systems, in Information Systems and e-Business Technologies: 2nd International United Information Systems Conference UNISCON 2008 Klagenfurt, Austria, April 22-25, 2008 Proceedings, R. Kaschek, et al., Editors. 2008, Springer Berlin Heidelberg: Berlin, Heidelberg. p. 175-188.

3. Hehenberger, P., et al., Design, modelling, simulation and integration of cyber physical systems: Methods and applications. Computers in Industry, 2016. 82: p. 273-289.

4. Seiger, R., S. Huber, and T. Schlegel, Toward an execution system for selfhealing workflows in cyber-physical systems. Software \& Systems Modeling, 2016: p. 1-22.

5. Schneider, C., A. Barker, and S. Dobson, A survey of self-healing systems frameworks. Software: Practice and Experience, 2015. 45(10): p. 1375-1398.

6. Loucopoulos, P. and V. Kavakli, Enterprise knowledge management and conceptual modelling, in Conceptual Modeling. 1999, Springer. p. 123-143.

7. Vernadat, F.B., Enterprise modelling and integration. 2003: Springer. 
8. Loucopoulos, P. and E. Kavakli, Enterprise modelling and the teleological approach to requirements engineering. International Journal of Cooperative Information Systems, 1995. 4(01): p. 45-79.

9. Robinson, S., Conceptual modelling for simulation Part I: definition and requirements. Journal of the operational research society, 2008. 59(3): p. 278-290.

10. Fayoumi, A. and P. Loucopoulos, Conceptual modeling for the design of intelligent and emergent information systems. Expert Systems with Applications, 2016. 59: p. 174-194.

11. Mylopoulos, J., Conceptual modelling and Telos 1. 2008, Citeseer.

12. Rolland, C. and N. Prakash, From conceptual modelling to requirements engineering. Annals of Software Engineering, 2000. 10(1-4): p. 151-176.

13. Schmidt, D.C., Model-driven engineering. COMPUTER-IEEE COMPUTER SOCIETY-, 2006. 39(2): p. 25.

14. Codd, E.F., A relational model of data for large shared data banks. Communications of the ACM, 1970. 13(6): p. 377-387.

15. Samavi, R., E. Yu, and T. Topaloglou, Strategic reasoning about business models: a conceptual modeling approach. Information Systems and eBusiness Management, 2009. 7(2): p. 171-198.

16. Hinkelmann, K., et al., A new paradigm for the continuous alignment of business and IT: Combining enterprise architecture modelling and enterprise ontology. Computers in Industry, 2015.

17. Singh, S.N. and C. Woo, Investigating business-IT alignment through multidisciplinary goal concepts. Requirements Engineering, 2009. 14(3): p. 177207.

18. Zachman, J.A., A framework for information systems architecture. IBM systems journal, 1987. 26(3): p. 276-292.

19. Zachman, J.A., Enterprise architecture: a framework. Zachman Institute, Colorado Spring, Colorado, USA, 2004.

20. TOGAF, The Open Group Architecture Framework (TOGAF) V. 9. The Open Group, 2009. 1.

21. Martin, J., An introduction to the Architectural Frameworks $D O D A F / M O D A F / N A F$. 2006, Course given at the Royal Institute of Technology, Stockholm, Sweden.

22. Frank, U., Domain-specific modeling languages: requirements analysis and design guidelines, in Domain Engineering. 2013, Springer. p. 133-157.

23. Laforcade, P., A Domain-Specific Modeling approach for supporting the specification of Visual Instructional Design Languages and the building of dedicated editors. Journal of Visual Languages \& Computing, 2010. 21(6): p. 347-358.

24. Loucopoulos, P., et al. Enterprise Capability Modeling: Concepts, Method, and Application. in 2015 International Conference on Enterprise Systems (ES). 2015. IEEE. 
25. Loucopoulos, P. and E. Kavakli, Capability Oriented Enterprise Knowledge Modeling: The CODEK Approach, in Domain-Specific Conceptual Modeling. 2016, Springer. p. 197-215.

26. Fill, H.-G., SeMFIS: a flexible engineering platform for semantic annotations of conceptual models. Semantic Web, 2017(Preprint): p. 1-17.

27. Bock, A. and U. Frank, Multi-perspective Enterprise Modeling-Conceptual Foundation and Implementation with ADOxx, in Domain-Specific Conceptual Modeling. 2016, Springer. p. 241-267.

28. Boissier, F., I. Rychkova, and J. Zdravkovic, Extending Enterprise Modeling for Decentralized Organizations. 2016, Université Paris 1-Panthéon Sorbonne; Stockholm University.

29. OMiLAB. Open Model Lab. [cited $201720 \mathrm{March}$ ]; Available from: http://www.OMiLAB.org/.

30. Karagiannis, D., et al., Fundamental Conceptual Modeling Languages in OMiLAB, in Domain-Specific Conceptual Modeling. 2016, Springer. p. 3-30.

31. Dietz, J.L., Enterprise ontology: theory and methodology. 2006: Springer Science \& Business Media.

32. Sandkuhl, K., et al., Enterprise Modeling. Tackling Business Challenges with the 4EM Method. Springer, 2014. 309.

33. Stirna, J., A. Persson, and L. Aggestam. Building knowledge repositories with enterprise modelling and patterns-from theory to practice. in ECIS 2006 PROCEEDINGS. 2006.

34. Coalition, W.M., BPSim standard. 2012.

35. Proudlove, N., et al., Towards fully-facilitated discrete event simulation modelling: Addressing the model coding stage. European Journal of Operational Research, 2017. 263(2): p. 583-595.

36. Kalenkova, A.A., et al., Process mining using BPMN: relating event logs and process models. Software \& Systems Modeling, 2017. 16(4): p. 10191048.

37. Stirna, J. and J. Zdravkovic, Interview with Sladjan Maras on" Challenges and Needs in Enterprise Modeling". Business \& Information Systems Engineering, 2015. 57(1): p. 79.

38. Bernaert, M., et al., CHOOSE: towards a metamodel for enterprise architecture in small and medium-sized enterprises. Information Systems Frontiers, 2015: p. 1-38.

39. Goul, M. and K. Corral, Enterprise model management and next generation decision support. Decision Support Systems, 2007. 43(3): p. 915-932.

40. Van Der Aalst, W.M.P., Process Mining: Discovery, Conformance and Enhancement of Business Processes. Springer-Verlag, 2011. 8: p. 18.

41. IBM. IBM Business Process Manager overview. 2018 [cited 2018 04/07]; Available from: https://www.ibm.com/support/knowledgecenter/SSFTBX_8.5.7/com.ibm.wb pm.main.doc/topics/ibmbmp_overview.html. 
42. Bădică, A., et al. Modeling and enactment of business agents using Jason. in Proceedings of the 9th Hellenic Conference on Artificial Intelligence. 2016. ACM.

43. Dalpiaz, F., P. Giorgini, and J. Mylopoulos, Adaptive socio-technical systems: a requirements-based approach. Requirements engineering, 2013. 18(1): p. 1-24.

44. Nachira, F., et al., Digital business ecosystems. 2007: European Commission, DG-Information Society and Media.

45. Peffers, K., et al., A design science research methodology for information systems research. Journal of management information systems, 2007. 24(3): p. 45-77.

46. Fill, H.-G. and D. Karagiannis, On the conceptualisation of modelling methods using the ADOxx meta modelling platform. Enterprise Modelling and Information Systems Architectures-An International Journal, 2013. 8(1).

47. Karagiannis, D. and N. Visic, Platform-as-a-Service (PaaS): The ADOxx Metamodelling Platform. 2011.

48. Eclipse. The Eclipse Modelling Framework official page. 2009 [cited 2017 March 20]; Available from: https://eclipse.org/modeling/emf/. 